Revue européenne des sciences sociales

European Journal of Social Sciences

52-1 | 2014

Politiques du libre accès en sciences humaines et sociales

\title{
Open access et SHS : Controverses
}

\section{Ghislaine Chartron}

\section{(2) OpenEdition}

Journals

Édition électronique

URL : http://journals.openedition.org/ress/2658

DOI : $10.4000 /$ ress. 2658

ISSN : 1663-4446

Éditeur

Librairie Droz

\section{Édition imprimée}

Date de publication : 6 mai 2014

Pagination : 37-63

ISBN : 978-2-600-01829-6

ISSN : 0048-8046

Référence électronique

Ghislaine Chartron, «Open access et SHS : Controverses », Revue européenne des sciences sociales [En ligne], 52-1 | 2014, mis en ligne le 12 mai 2014, consulté le 03 mai 2019. URL : http://

journals.openedition.org/ress/2658; DOI : 10.4000/ress.2658 


\title{
OPEN ACCESS ET SHS : CONTROVERSES
}

GHISLAINE CHARTRON

CNAM-DICEN-IdF

ghislaine.chartron@cnam.fr

Résumé. Après avoir rappelé la genèse du mouvement pour l'Open Access et fait état de la vivacité des débats actuels autour du sujet, cet article s'attache à discuter le bien-fondé de l'injonction politique en faveur du libre accès aux publications scientifiques au regard des spécificités de la recherche en sciences humaines et sociales. Partant du constat que les politiques publiques s'élaborent majoritairement selon des caractéristiques empruntées aux sciences biomédicales, technologiques ou de la nature, l'auteur questionne la pertinence des postulats avancés, notamment la barrière d'accès aux savoirs, et examine les enjeux en matière d'innovation, de croissance et de retour sur l'investissement public, en particulier dans la perspective des données ouvertes et du datamining. L'article souligne les risques d'une transformation numérique non mesurée pour l'édition des sciences humaines et sociales et esquisse différents scénarios possibles selon les politiques publiques qui auront été décidées.

Mots-clés: communication scientifique, libre accès, politiques publiques, revues scientifiques, sciences humaines et sociales.

\begin{abstract}
After dealing with the genesis of the Open Access movement and reporting the current controversial debates on the subject, this article aims to discuss the political injunction towards the open access to scientific publications considering the characteristics of the Human and Social Sciences field. On the principle that these public policies are mostly based on features that derived from the techical, biomedical or natural sciences, the author challenges the pertinence of some of the common assumptions, namely the barrier to access of knowledge, and examines the stakes in regard to innovation, growth and return of investment, particularly in the prospect of open data and datamining. The article stresses the possible impact that the digital transformation may have on publishing in the field of human and social sciences and outlines different scenarios depending on the public policies implemented.
\end{abstract}

Keywords: academic journals, human and social sciences, open access, public policy, scholarly communication. 
INTRODUCTION

Peut-on être contre l'Open Access (OA) ? Qui serait contre l'idée de pouvoir accéder librement à toutes les connaissances disponibles ? Cette vision nous rapproche inévitablement de la maxime fondatrice de Google qui, par ailleurs, en début 20I4, vient d'être classé comme la deuxième capitalisation boursière mondiale, derrière Apple... Philanthropie? Idéologie utopique? Sacrifice des acteurs producteurs de contenu au profit des acteurs de la technologie? À qui profite l'OA ? Concernant le domaine de l'édition scientifique, le débat n'est-il pas devenu illisible au fil des années avec aujourd'hui un centrage sur la transparence de l'action publique, une croyance en l'innovation et en une reprise de croissance dont une des sources serait le libre accès aux publications scientifiques?

Une certaine prise de recul est nécessaire afin de mesurer les risques que l'impératif du libre accès peut faire courir aux équilibres et aux dynamiques en place dans la production éditoriale de nombreux champs scientifiques, somme toute de qualité, diversifiée et intéressante. Il serait hasardeux de penser les transformations numériques sans prendre la mesure fine des différents contextes de production, car tout retour arrière sera difficile. Innover dans la communication scientifique est un vrai défi pour améliorer la qualité du processus, mais faire table rase de l'existant au nom de l'innovation est un pari risqué.

Telle sera la posture débattue dans cette contribution, partant de l'actualité d'une injonction politique pressante, notamment américaine et européenne, qui semble ne pas vouloir prendre le temps de la mesure et de la concertation avec les chercheurs, à l'aune de la diversité du travail intellectuel qu'ils accomplissent, ni avec les éditeurs, considérant la diversité de leurs caractéristiques structurelles. L'opinion «mainstream» qui tend à s'exprimer aujourd'hui est plutôt simplificatrice et risque même de s'avérer destructrice. Le débat est majoritairement alimenté par les représentants des sciences bio-médicales ou des sciences de la nature avec des arguments convaincants mais qui ne peuvent être transposés sans précaution à tous les domaines scientifiques et notamment en sciences humaines et sociales (SHS). Nous voudrions en expliciter la 
raison. L'hypothèse d'un patron unique dans lequel devraient se mouler tous les champs scientifiques, telle qu'elle est formulée par une technocratie imprudente, est une hypothèse qui pourrait être fatale à des pans entiers de l'édition.

\section{OPEN ACCESS, MISE EN PERSPECTIVE ET ACTUALITÉS}

\section{I.I. L'ESSENTIEL}

L'histoire de l'OA commence au début des années 1990, si l'on se réfère à l'ouverture pionnière de l'archive ouverte de prépublications ArXiv, en I99I par Paul Ginsparg et pour la communauté des physiciens théoriciens. Le projet est porté par les chercheurs, inscrit dans une pratique commune d'échanges des papiers entre laboratoires par courrier postal. L'innovation connut très vite un vif succès, greffée sur une pratique sociale antérieure au dispositif numérique qui améliorait considérablement le service rendu. Au croisement de l'Internet et de la communication scientifique, les années 2000 ont ainsi vu naître de très nombreuses initiatives dont il serait intéressant de s'assurer de la pérennité aujourd'hui... L'OA a connu des phases différentes, les enjeux n'ayant cessé d'être reformulés par différents groupes d'acteurs dont la vision tend aujourd'hui à ne plus converger véritablement. Le mouvement fut d’abord le fait des chercheurs pionniers et visionnaires, à l'instar de Paul Ginsparg, Stevan Harnad (I990) ou Andrew Odlzysko (1995), puis inspira la révolte des bibliothèques de recherche (voir notamment Okerson, O’Donnel, 1995) face à l'augmentation des prix de certaines revues en sciences biomédicales et en sciences dures et donna naissance à la coalition SPARC (Scholarly Publishing and Academic Resources Coalition). En parallèle des déclarations militantes internationales ${ }^{1}$ ont contribué à populariser la thématique de l’OA, à la suite de la lettre ouverte émise par la Public Library of Science (PLOS) à l'occasion de sa fondation en 200I, dont celle de Budapest en 2002 (inaugurant la thématique des deux «voies» du libre accès), ou celle de Berlin en 2003 (soutenue par la société Max-Planck). Ces dernières années ont vu la reprise en main du mouvement par les acteurs politiques, l'Office of

I Les textes de référence sont accessibles sur le blog de l'INIST: <http://openaccess.inist.fr/?Textes-de-references->. 
Science and Technology Policy aux États-Unis, et la Commission européenne (CE) qui associe à l'OA une vision très libérale de soutien à l'innovation : les résultats de la recherche publique doivent irriguer le monde socio-économique et favoriser son développement. Cette vision est également portée par l'OCDE dans ses différents rapports depuis plusieurs années (OCDE, 2007, 2012), sources d'inspiration majeures des politiques économiques européennes. Le dernier rapport insiste sur les différentes modalités de commercialisation de la recherche et des productions diverses des chercheurs (OCDE, 2013).

L’OA est-il devenu illisible au fil du temps ? La question se pose tant les différents acteurs concernés déploient désormais des arguments qui peuvent profondément s'opposer. La vision très libérale des politiques européennes semble, par exemple, bien loin de l'idéologie des savoirs-communs de la connaissance défendue par des groupes de chercheurs et des bibliothèques militantes, dans une veine libertaire. De la même façon, la conception de l'OA comme outil de mesure de la production scientifique au travers des dépôts effectués dans les archives ouvertes semble bien éloignée de la motivation des chercheurs pionniers œuvrant pour la communication scientifique sans frontière. Enfin, les bibliothèques qui militent pour les mandats obligatoires ou qui privilégient certains opérateurs ne convergent pas vraiment avec la posture de la majorité des chercheurs qu'elles sont censées représenter... Le paysage est donc un peu brouillé.

\section{I.2. ACTUALITÉS VIVES}

Le gouvernement américain et la CE sont aujourd'hui les deux acteurs majeurs qui programment l'agenda de l'OA et ses modalités de développement. Aux États-Unis, le discours est essentiellement axé sur la transparence de l'investissement public dans la perspective d'un retour de cet investissement pour les contribuables. La loi de finances 20I4 (FY 20I4 Omnibus Appropriations Bill $^{2}$ ) inscrit désormais le dépôt obligatoire des articles de chercheurs dépendant des agences de trois ministères (Travail, Santé et Éducation) lorsque leur financement global dépasse les ıoo millions de dollars annuels. La version 
finale des articles, résultant de recherches financées pour tout ou partie devra être accessible librement dans les 12 mois suivant la date de publication. Cette mesure étend l'obligation de dépôt de la politique pionnière de l'agence de santé, National Institutes of Health, fixée en $2008^{3}$.

L’agenda européen, quant à lui, a été fixé par la CE lors de la publication de deux Recommandations le I7 juillet 2012 : l'une ${ }^{4}$ concernant les politiques nationales de chaque État membre, la CE affirmant son soutien aux deux voies ${ }^{5}$ de l'OA et recommandant des délais d'embargos de 6 mois en sciences techniques et médicales (STM) et de 12 mois en SHS au maximum. L'objectif est de parvenir à $60 \%$ de publications issues de recherches financées sur fond public disponibles en libre accès en 2016, 100\% en 2020. L'autre ${ }^{6}$ concerne, de façon liée, le développement de l'espace européen de la recherche qui doit s'adosser à des logiques d'innovation ouverte, de mobilité des chercheurs et de transferts public-privé. Dans ce cadre, l'accès ouvert aux publications est considéré comme une brique essentielle. Début 20I4, l’Open Data (données ouvertes de la recherche) est devenu l'actualité majeure? ${ }^{7}$ nous reviendrons sur ces enjeux par la suite.

Le gouvernement britannique est le troisième acteur le plus influent sur le développement de l’OA. Le rapport de Janet Finch ${ }^{8}$ (I8 juin 20I2), «Accessibility, sustainability, excellence: how to expand access to research publications» a privilégié la voie dorée (Gold OA). David Willets, Ministre de l'enseignement supérieur et de la

3 Voir <http://publicaccess.nih.gov/>.

4 Voir <http://ec.europa.eu/research/science-society/document_library/pdf_06/era-communication-towards-better-access-to-scientific-information_en.pdf>.

5 Pour rappel, on considère que la voie dorée (Gold $\mathrm{OA}$ ) correspond à la publication dans des revues en libre accès immédiat, en général suivant la modalité de l'auteur-payeur, et la voie verte (Green OA) au dépôt par leurs auteurs d'une version de leur article dans les archives ouvertes, après un délai éventuel de rétention de durée limitée.

6 Voir <http://ec.europa.eu/research/science-society/document_library/pdf_06/era-communication-partnership-excellence-growth_en.pdf>.

7 Voir Guidelines on Open Access to Scientific Publications and Research Data in Horizon 2020: <http:// ec.europa.eu/research/participants/data/ref/h2020/grants_manual/hi/oa_pilot/h2020-hi-oa-pilot-guide_en.pdf> ; Guidelines on Data Management in Horizon 2020: <http://ec.europa.eu/research/participants/data/ref/h2020/grants_manual/hi/oa_pilot/h2020-hi-oa-data-mgt_en.pdf>.

8 Voir <http://www.researchinfonet.org/wp-content/uploads/2012/06/finch-group-report-final-version.pdf>. 
recherche a suivi ce modèle et fixé un plan de financement supplémentaire de IOM£ en 20I4, tout en reconnaissant aussi la voie verte (Green OA) en second choix avec des embargos de 6 ou I 2 mois. Le constat actuel est plutôt celui d'une difficile transition, les universités ne disposant pas de fonds suffisants pour soutenir la voie dorée. Les sociétés savantes très actives ont contesté les délais d'embargo arbitrairement décidés. Une période de test est envisagée pour les 5 ans à venir avec d'éventuelles révisions des politiques initialement énoncées.

Le gouvernement français, quant à lui, par le discours du 24 janvier $2012^{9}$ de Mme Fioraso, Ministre de l’Enseignement supérieur et de la Recherche, affirme son soutien à l'OA selon des modalités très variées en accord avec les communautés scientifiques. La politique nationale devrait se préciser en 20I4, une étude étant notamment en cours afin de mesurer les impacts pour les revues de $\mathrm{SHS}^{\mathrm{I}}$.

\section{I.3. L'EXPRESSION D'UN DÉSACCORD}

Ces politiques d'OA, empreinte d'une croyance affirmée pour l'innovation, ont généralement peu mesuré les effets collatéraux sur les acteurs de l'édition. La création de valeur attendue en aval de ce type de mesure tend à sacrifier sans discernement la créativité en amont de la fonction éditoriale. Le débat tend à se focaliser uniquement sur les dérives des grands groupes internationaux de l'édition en STM et oublier la fragilité des modèles économiques des plus petits éditeurs. Progressivement, les réactions ont émergé dans les pays où un tissu éditorial national opère de façon significative pour l'édition de la recherche. Au Royaume-Uni, la politique de l'OA a été perçue comme particulièrement agressive ainsi qu'en France où de nombreux éditeurs SHS ont retrouvé une dynamique récente pour le numérique autour de la plateforme Cairn.info, notamment.

9 Voir <http://www.enseignementsup-recherche.gouv.fr/cid66992/discours-de-genevieve-fioraso-lors-des-5e-journees-open-access.html>.

I0 Voir<http://www.ipp.eu/fr/thematiques/politiques-sectorielles/revues-de-sciences-humaines-et-sociales-shs-impact-politique-de-libre-acces/>. 
Deux associations d'éditeurs britanniques, l'Association of Learned, Professional and Society Publishers (ALPSP) et The Publishers Association, publiaient en 2012 les résultats d'une enquête auprès d'un échantillon de 2 Io bibliothèques à l'international, l'objectif étant de mesurer l'impact potentiel d'une politique d'OA avec un embargo de 6 mois sur les désabonnements des bibliothèques (ALPSP, 20ı2). La question posée était la suivante : si une revue est en accès libre 6 mois après sa publication, continueriez-vous à vous abonner? Une réponse était demandée pour chacun des domaines STM et SHS. Globalement, l'enquête a montré que les désabonnements seraient importants et que la mesure serait particulièrement dommageable pour la diffusion des SHS, à l'endroit desquelles seulement $35 \%$ des bibliothèques déclaraient qu'elles conserveraient les abonnements dans leur totalité (contre un taux de $56 \%$ pour les STM).

Au Royaume-Uni, les historiens ont affiché leur opposition aux directives : 2 I éditeurs en chef de revue, dans une lettre ouverte ${ }^{\text {II }}$ (décembre 2012) ont spécifié que l'embargo devait être au minimum de 36 mois et que la qualité devait rester le critère majeur de la publication. Des réactions continuent à se faire jour dans cette communauté qui considère les éditeurs comme des partenaires et qui revendique la responsabilité de décider des modalités viables pour la transformation pour leurs éditions ${ }^{12}$. De la même façon, la British Academy, qui assure la promotion des SHS au Royaume-Uni, a réagi vivement à cette injonction du gouvernement et publié une contribution majeure de plusieurs chercheurs et éditeurs académiques argumentant dans le sens d'une limitation de l'OA sur le terrain des SHS (Vincent, Wickham, 20I3).

En France, ce sont les comités de rédaction de i27 revues qui se sont mobilisés en adressant une lettre ouverte (Collectif, 20ı3) à la Ministre de l'Enseignement supérieur et de la Recherche, à la Ministre de la Culture et de la Communication et aux présidents des universités et des grandes écoles ainsi qu'aux responsables des grands établissements de recherche:

II Voir <http://www.history.ac.uk/news/2012-12-10/statement-position-relation-open-access>.

12 Voir <http://www.insidehighered.com/news/2014/01/06/historians-clash-over-open-accessmovement\#.usrdsjkhbry.email>. 
Nous appelons à l'ouverture rapide d'une véritable concertation sur les enjeux de l'Open Access en sciences humaines et sociales; la définition de périodes d'embargo suffisantes, permettant aux revues de choisir leur modèle économique (par exemple, en équilibrant le gratuit et le payant), est l'unique garantie de la diversité et de l'indépendance de la recherche scientifique et du débat public (ibid.).

L’inquiétude est palpable et les gouvernements ont donc été interpellés pour mesurer leur politique au regard d’effets collatéraux qui seraient irréversibles.

\section{RETOUR SUR LA COMMUNICATION ET L'ÉDITION ACADÉMIQUE EN SHS}

Les politiques publiques pour l'OA se préoccupent dans l'ensemble assez peu des différences structurelles de la communication scientifique selon les communautés. De telles différences sont pourtant fondamentales et il conviendrait de les considérer attentivement avant toute décision susceptible d’affecter un processus qui n'est pas homogène selon les communautés scientifiques.

\section{I. TRAVAIL INTELLECTUEL ET MODE DE PUBLICATION}

La communication scientifique porte les caractéristiques du travail intellectuel produit ainsi que les caractéristiques d'échange de la communauté concernée. Sur ces deux points, STM et SHS ne convergent pas. Toute politique en matière d'OA devrait en tenir compte. Nous voudrions insister sur ce point car la focalisation actuelle sur les articles des revues par les recommandations politiques traduit une simplification du projet, inspirée des STM.

Prenons l'exemple de l'histoire médiévale que nous avions déjà investiguée (Chartron, 2003), domaine dans lequel la publication de monographies revêt un caractère supérieur à celle d’articles scientifiques. L'écriture fait partie du processus de création de la connaissance pour l'historien, elle permet la formalisation de la problématique et la mise en place de l'argumentation après l'étude des sources, travail préalable à toute étude historique. En cela, nous pouvons les opposer à certaines sciences dures pour lesquelles l'écriture est le moment de la diffusion de la connaissance créée au préalable par l'expérience en laboratoire. Cette particularité a des conséquences sur la place des différents types 
de publications dans l'information historique. Ainsi, la monographie occupe la place la plus importante. Elle représente le travail préalable à toute autre diffusion du travail historique. La publication d’articles a lieu après ce passage obligé de l'écriture d'une monographie. Sa carrière de chercheur est souvent consacrée lorsqu'il est sollicité par un éditeur grand public (Seuil, Fayard ou Gallimard, par exemple) dans le cadre de collections particulières de son catalogue. De la même façon, une récente enquête menée par l'INSHS du CNRS sur une population de 1762 chercheurs CNRS, confirme de façon factuelle la place des ouvrages et des chapitres d'ouvrages dans la communication en SHS :

Le traitement des données à partir de RiBAC montre que les ouvrages, chapitres d'ouvrages et directions d'ouvrages représentent près de $45 \%$ des publications totales des chercheurs en SHS. Les livres constituent donc, pour les sciences humaines et sociales, un support majoritaire de diffusion des connaissances, une particularité qui est propre à cette communauté. Comme pour les revues, la publication sous forme d'ouvrages varie selon les disciplines des sciences humaines et sociales; ainsi les historiens, philosophes et anthropologues publient deux fois plus de chapitres d'ouvrages que les chercheurs en économie qui eux privilégient la diffusion de leurs travaux dans des revues ${ }^{13}$.

Contrairement aux STM, les revues SHS ne représentent donc pas toujours l'excellence scientifique, elles ne sont pas ces lieux incontournables de confrontation internationale de la recherche comme elles le sont pour les STM ${ }^{14}$. S’il faut identifier et diffuser l'excellence scientifique, il faut alors d'abord considérer les monographies pour les $\mathrm{SHS}^{15}$, comme les actes de colloques pour les sciences de l'ingénieur par ailleurs.

13 Voir «Les ouvrages scientifiques en sciences humaines et sociales - Résultats de l'enquête RIBAC 20 II 》, sur une population de 1762 chercheurs CNRS-SHS, lettre de I'INSHS/CNRS, juillet 2013: <http://www.cnrs.fr/inshs/lettres-information-inshs/lettre_info24.pdf>.

14 Au sein même des champs STM, R. Kling et G. Mckim (2000) avaient aussi revisité les différences.

I5 On notera par exemple, que la Fondation nationale pour l'enseignement de la gestion des entreprises (FNEGE) a introduit, parallèlement à son classement des revues, une labellisation des ouvrages: <http://www.fnege.net/publications_evaluation/62-fr-college_de_labellisation_des_ouvrages> ; <http://www.fnege.net/Liens_utiles_Ressources/l00-FR-Classement_des_Revues_Scientifiques_de_Gestion>. 


\subsection{CARACTÉRISTIQUES STRUCTURELLES DES REVUES EN SHS}

Une autre considération importante concerne le type d’acteurs en charge de l'édition des champs scientifiques et notamment des revues dont il est principalement question. Les revues SHS sont avant tout le vecteur de communication d'équipes de recherche et d'écoles de pensée différentes. Il en résulte un extrême morcellement de l'offre de revues SHS, source de diversité et d'opportunités de publication pour les chercheurs, mais source également de difficiles confrontations intellectuelles entre chapelles, il est vrai. Comme l'ont montré des travaux antérieurs (GFII, 2009), les acteurs en charge de l'organisation de la publication et de la diffusion sont très divers, mais une majorité d'entre eux opèrent au niveau national, contrairement aux champs STM. La publication dans la langue nationale reste importante et les éditeurs se répartissent entre éditeurs commerciaux, société savantes, laboratoires, presses universitaires, associations, institutions publiques, etc. Si l'on veut caractériser les acteurs suivant une logique «acteur purement public » ou non, la répartition est aussi très différente selon les pays et l'histoire respective des entrepreneurs éditoriaux nationaux. Dans l'étude comparative que nous avons menée avec Marc Minon (Chartron, Minon, 2005), cette différence avait été pointée, l’Espagne étant caractérisée par une prépondérance d'acteurs universitaires, l'Italie par l'activité dominante d’acteurs privés et la France par une dualité partagée entre des maisons d'édition nationales, d'origine familiale (Armand Colin, Lavoisier, PUF, L’Harmatan, Eska, Érès, Le Seuil, etc.) et une diversité d'acteurs publics dont principalement les presses universitaires et les organismes de recherche. Toute politique d'OA en SHS devra mesurer son impact sur ces acteurs de l'édition nationale. Contrairement aux STM, le périmètre de développement de leurs activités est plus restreint et les équilibres économiques plus fragiles entre activités éditoriales et autres activités. 
La notoriété dans ces domaines se construit également sur des publications à lectorat élargi que les maisons d'édition visent à développer en sélectionnant leurs auteurs et en élaborant un catalogue bien identifiable. Il est ainsi toujours difficile de fixer le périmètre des revues de recherche ${ }^{16}$ lorsqu'il s'agit des SHS tant leur lectorat est diffus, signalant d’ailleurs la reconnaissance de leur valeur par-delà le cercle des spécialistes. A contrario, «les chercheurs parlent aux chercheurs » est manifestement le contrat d'écriture-lecture pour les STM dans la progression d'une connaissance très pointue souvent mathématisée. Pour les SHS, même si des travaux pointus prennent place également dans des revues spécialisées, d’autres types de production relèvent plus d’une analyse critique, du développement d'une pensée personnelle dont l'expression intéresse aussi le grand public et le public professionnel. Le transfert sciences-société se fera ainsi davantage par des revues de lectorat hybride plus que par l'OA dans bien des cas. Comparons par exemple une revue comme Le Débat et une revue scientifique pointue d'une presse universitaire locale: quelle est celle qui, manifestement, aura le plus d’impact sur la société au sens des enjeux exposés par l'OA?

\section{INTERROGER LES POSTULATS DE L'OA AU REGARD DES SHS}

Revenons désormais sur certains arguments majeurs mis en avant aujourd'hui pour soutenir une politique de libre accès aux publications scientifiques. L'hypothèse défendue est que ces arguments sont forgés principalement pour des domaines inscrits dans la recherche de pointe, en phase avec des enjeux de compétitivité internationale, de développements économiques (biologie, biotechnologie, neurosciences, énergies renouvelables, environnement, vieillissement...). Les arguments politiques sont généralement énoncés comme des postulats, qui permettent de justifier la vision déployée. Il convient de revenir sur ces postulats lorsqu'ils veulent aussi s'appliquer aux SHS car la légitimation est souvent peu recevable. 


\section{I. L'INFLATION DES COÛTS DES PUBLICATIONS ET LA BARRIÈRE D'ACCÈS AUX SAVOIRS}

L'inflation des coûts des revues STM dans les années 2000, notamment celles d'éditeurs commerciaux internationaux, fut l'une des origines majeures du mouvement pour l'OA porté initialement par les bibliothèques de recherche, à majorité américaines. Cette inflation a été largement documentée par les statistiques annuelles de l'Association of Research Libraries mettant en évidence la croissance exponentielle des dépenses consacrées aux revues et l'écart grandissant de ces dépenses au regard de celles réservées aux ouvrages. Les dernières statistiques $^{17}$ montrent une augmentation des dépenses des périodiques de + $402 \%$ ces 25 dernières années alors que l’augmentation n’a été que de + 7r \% pour les monographies sur la même échelle de temps. Plus précisément, une étude économétrique de la CE (2006) avait comparé le prix des revues en STM selon les éditeurs commerciaux internationaux (64\%) et les éditeurs non commerciaux (essentiellement des sociétés savantes) : «les revues d'éditeurs commerciaux seraient 2,7 à 3 fois plus chères que les autres revues pour un domaine, un âge et un nombre de citations».

Une revue en STM coûte généralement entre 400 et 2500 euros par an ${ }^{18}$ avec quelques pics vertigineux comme la revue Brain research, par exemple, dont l'abonnement annuel à la version papier atteint I7 800 euros en 20I4. En comparaison, le tarif des revues internationales en SHS est en moyenne de l'ordre de 370 euros, selon les données rassemblées dans l'étude du GFII (2009). Ces comparaisons brutes doivent certes être nuancées par la fréquence de parution des revues: il est habituel qu'une revue en SHS soit semestrielle tandis qu'une revue en STM est plus souvent mensuelle. Toutefois, quand on considère le prix des revues nationales en SHS, l'écart des prix devient alors très significatif. Selon la même étude du GFII, le coût moyen de l'abonnement était de 220 euros par an pour les revues françaises et seules ıo revues de l'échantillon étudié (soit 6,58\%) affichaient un prix de l’abonnement annuel supérieur à I50 euros (voir Figure I).

17 Statistiques ARL: <http://www.arl.org/storage/documents/monograph-serial-costs.pdf>.

I8 Voir par exemple les tarifs de Springer: <http://www.springer.com/librarians/>. 
Figure I : Prix des revues SHS en euros d'éditeurs de revues françaises

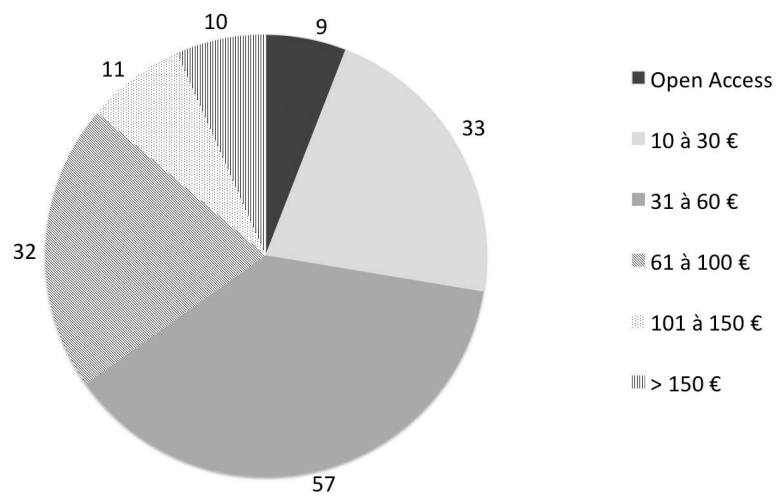

Source: Étude GFII-SHS, 2009, vol.3, p.7

Avec de tels tarifs, on ne peut alors pas parler d'inflation vertigineuse des coûts, ni de barrière d'accès pour les bibliothèques universitaires françaises. On peut en revanche évoquer l'inflation du nombre de revues nationales. Il est à cet égard de la responsabilité des politiques d'acquisition de fixer des priorités en fonction de la qualité des contenus proposés et de l'intérêt porté par les lecteurs.

Le réel problème pour les SHS réside dans l’absorption de la majorité du budget des bibliothèques par l'abonnement aux revues en STM. Par ailleurs, les connaissances s'accumulant, les champs se diversifiant et les chercheurs et les étudiants étant toujours plus nombreux, les dépenses peuvent difficilement décroître. Raisonner uniquement avec le budget fléché pour les bibliothèques n'est certainement plus tenable, il faut désormais mobiliser les crédits de la recherche pour la documentation de recherche et consacrer le budget des bibliothèques prioritairement aux étudiants. En France, le budget des universités ne faiblit pas $^{19}$. Il faut donc raisonner sur la dotation globale des établissements : pourquoi ne pas rendre prioritaire le budget de cette fonction centrale que repré-

19 «Les dotations des universités et des établissements d'enseignement supérieur progressent entre $0,15 \%$ et 3,0\% par rapport à l'année dernière», a annoncé Geneviève Fioraso vendredi 13 décembre 2013: <http://www.letudiant.fr/educpros/actualite/budget-des-universites-larepartition-des-dotations-20|4.html>. 
sente l'accès à des ressources de qualité permettant notamment des processus complémentaires d’apprentissage ? Pourquoi les établissements tendent à réduire en priorité le budget des ressources documentaires alors que les dérapages les plus importants s'observent souvent sur la gestion de la masse salariale?

\subsection{ENJEUX SUR L'INNOVATION ET LA CROISSANCE}

Le libre accès à la connaissance est adossé par ailleurs à un paradigme souvent mis en avant dans l'économie numérique, à savoir l'open innovation qui postule que, pour être créatif et répondre aux défis contemporains, il est désormais plus efficace de ne pas se fonder uniquement sur sa propre recherche: il faut coopérer (Chesbrough, 2003; Penin, 20II). L'innovation ouverte modifie ainsi les frontières de l'entreprise, elle substitue au schéma de la R\&D interne fermée un schéma de créativité collaborative et partenariale en phase avec la complexité des nombreux problèmes à résoudre. On pourrait douter de ce schéma quand on constate le succès de sociétés comme Apple et Google dont la stratégie est majoritairement inverse, avec une $R \& D$ qui reste confinée en interne (après rachat des talents) et protégée par une multitude de brevets ${ }^{20}$. Quoiqu'il en soit, dans cette vision, la collaboration recherche-entreprise est un axe majeur défendu pour la croissance. Ses modalités de développement font l'objet de comparaisons publiées par l'OCDE (20I3). Pour le débat qui nous intéresse, une tension peut notamment s'exercer entre la promotion simultanée d'une politique soutenue de dépôts de brevets et d'une politique de libre accès. Laquelle de ces politiques est-elle la plus favorable à la rencontre partenariale public-privé ?

Par ailleurs, est-il vraiment raisonnable de considérer que la recherche en SHS présente autant d'enjeux sur la relance économique et la création d’emplois que la recherche sur le génome, les nouveaux matériaux, les neurosciences, les énergies renouvelables, etc. ? Il est permis d'en douter sans pour

20 Plus de 4000 brevets déposés par Google: <http://patft.uspto.gov/netacgi/nph-Parser?Sect I=PTO2\&Sect2=HITOFF\&u=\%2Fnetahtml\%2FPTO\%2Fsearch-adv.htm\& $r=0 \& f=S \& \mid=50 \& d$ =PTXT\&RS=AN\%2Fgoogle\&Query=AN\%2Fgoogle\&TD=689\&Srchl=google.ASNM.\&Sta rtAt=Jump+To\&StartAt=Jump+To\&StartNum $=\mid>$. 
autant sous-estimer la valeur des travaux en SHS, dont l'intérêt réside beaucoup plus dans les grilles d'analyses qu'elles nous livrent pour comprendre le monde, les organisations humaines et leurs évolutions. Certains défendent alors, pour cette raison même, l'accessibilité ouverte à ces textes en vertu d’enjeux centrés désormais sur la citoyenneté, l'éducation politique, etc. L'auteur en SHS est, de notre point de vue, plutôt enclin à s'y opposer car sa production relève très souvent d'une production intellectuelle individuelle importante, à la différence des articles co-signés par 50 collaborateurs en astrophysique, par exemple. L'ouverture des textes ne doit pas non plus conduire à remettre en cause l'existence même des revues en contribuant à la fragilisation des équilibres de fonctionnement trouvés.

\subsection{LE RETOUR SUR INVESTISSEMENT PUBLIC (ROI)}

Un autre argument fréquemment avancé pour justifier l'OA est celui du retour sur investissement mis en avant par la CE, au nom de la transparence de l'investissement communautaire sur la recherche et l'innovation. Ce raisonnement s'inscrit dans l'orientation dominante depuis plusieurs années en matière de pilotage des politiques publiques qui met l'accent sur la performance. La performance est un ratio théoriquement simple à énoncer :

(Gain de l'investissement - coût de l'investissement) / Coût de l'investissement

La difficulté du calcul réside de facto dans l'appréciation du gain de l'investissement: tout le défi est de trouver des indicateurs qui puissent l'éclairer. Dans le cas de la recherche, le comptage et l'accès aux publications issues de recherches financées sur fonds publics sont des indicateurs qui se sont imposés. On pourrait les discuter au regard d'autres indicateurs envisageables comme la création d'entreprises, le dépôt de brevets, le développement de la réflexivité personnelle, etc.

L'examen de l'investissement communautaire selon les différents domaines scientifiques (voir Figure 2) montre que les SHS (ici SSH, en anglais) ne représentent en fait, en 20I3, qu’une très faible partie des financements sur projets (environ $3 \%$ ). 
Figure 2: Répartition du financement de projets de la CE par thématique en 2013

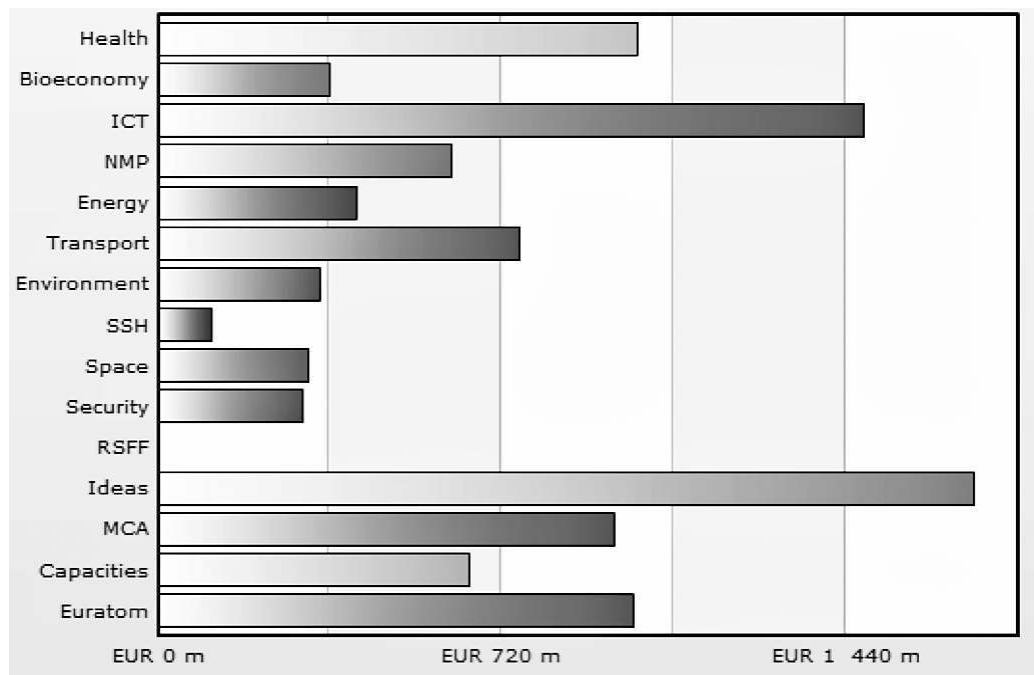

Source: Commission européenne, 30 years of EU investment in Research and Innovation: $<$ http://ec.europa.eu/research/fp7/index_en.cfm?pg =budget>.

On pourrait alors penser que la recherche de financements dans ces domaines se formule davantage au niveau national et que le pourcentage est donc plus élevé dans l'examen des bilans de l'ANR pour la France, par exemple. Or, il n'en est rien (voir Figure 3) : en 20I2, les SHS ne couvraient que 2,2 \% des dotations attribuées au titre des appels à projets de l'ANR. 
Figure 3 : Répartition du financement de projets de l'ANR par thématique en 2012

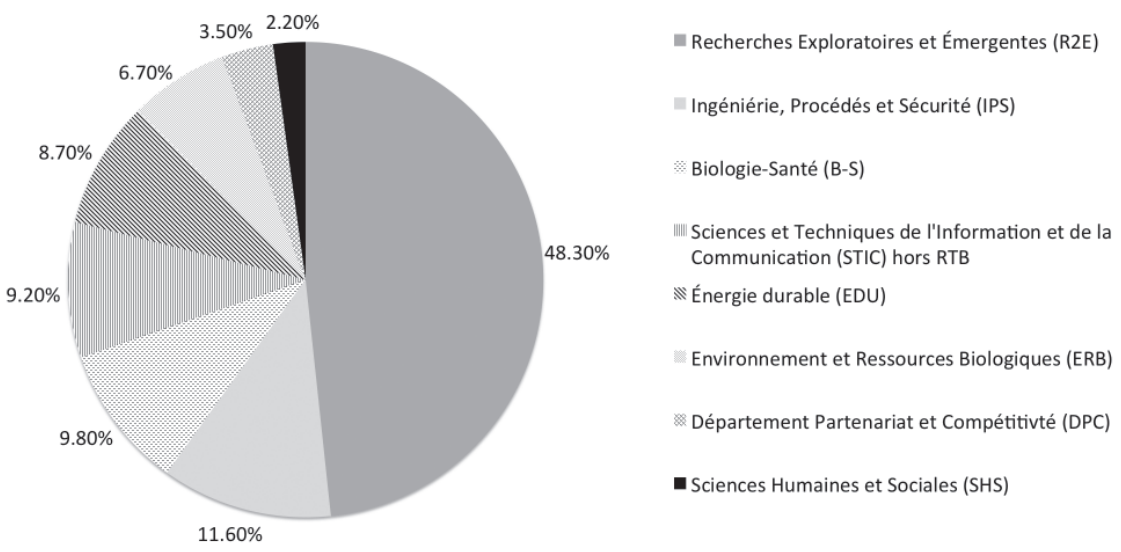

Source: <http://www.agence-nationale-recherche.fr/fileadmin/documents/20 I3/ANR-rapport-annuel-20|2.pdf>.

S’il faut donc justifier un ROI pour la recherche, il faut prioritairement le chercher dans des domaines captant l'essentiel des subventions publiques de recherche, à savoir l'ingénierie, la biologie, les STIC, l'énergie, l'environnement, la santé, etc. En examinant le montant des financements distribués, on peut aussi comprendre pourquoi les délais d'embargo soient voulus les plus courts possibles dans certains domaines. Il est par contre déraisonnable de vouloir appliquer sans discernement de tels délais, valables pour les STM, aux SHS, car cela pourrait conduire à la destruction de leur valeur spécifique, à savoir leur diversité et richesse éditoriales.

Penser, par ailleurs, que la performance de la recherche en SHS dépend essentiellement de financements de projets est aussi une erreur d'appréciation. Comparé aux STM, le coût de l'«appareillage» de la recherche est très faible. On ne peut pas tirer argument du ROI de la même façon. On pourrait en revanche - et l'argument serait juste - invoquer les salaires de ces chercheurs comme un investissement majeur. Mais, en terme de «productivité » et d'«efficience», ne vaut-il pas mieux veiller à l'indépendance de la production intellectuelle, en garantissant la liberté de publication selon des régimes 
qui stimulent les chercheurs, au travers de partenariats internes et externes aux communautés? Et cela plutôt que de fragiliser les modalités éditoriales en imposant des modes de fonctionnements incertains, pouvant finalement réduire le champ des possibles?

\subsection{L'OPEN DATA ET LE DATAMINING}

L’actualité du libre accès aux données de la recherche est une autre étape de l'Open Science (Royal Society, 20I2), dont les enjeux ont été développés dans un rapport de l'OCDE (2007). Les bases de données numériques sont désormais considérées comme un élément essentiel de l'infrastructure du système scientifique mondial. Le rapport insiste sur l'efficience de l'investissement dans la recherche, l'efficience de la recherche par la capitalisation et le partage des résultats, l'enjeu des croisements disciplinaires. Le modèle emblématique est celui du génome humain, il est cité dans la majorité des communiqués politiques en faveur de l'Open Data. L'actuel projet ARGO nous fournit un autre exemple : c'est un projet international d'observation de l'océan disposant d'un réseau de 3000 flotteurs autonomes, répartis sur l'ensemble des océans, qui mesurent la température et la salinité depuis la surface jusqu'à 2000 mètres de profondeur. Les données collectées par ces flotteurs sont diffusées en quasi temps réel (après un ensemble de contrôles automatiques de qualité). Les données sont disponibles librement et sans restriction.

Dans ces deux exemples et conformément à la vision de l'OCDE, il s'agit de données brutes collectées par des appareils de séquençage ou de mesure et qui peuvent être utilisées par tous les chercheurs travaillant dans le domaine concerné. L’astrophysique et la sismologie partagent de façon analogue des appareils internationaux de mesure de phénomènes naturels. Ces données font l’objet d'identification unique et stable afin de pouvoir, notamment, être citées dans les publications scientifiques. Éditeurs et communautés dialoguent actuellement en vue d'une optimisation de l'accès aux données. Un autre enjeu majeur consiste dans la fouille de données (datamining), à savoir le traitement statistique de ces masses de données pour en faire émerger d'inédites corrélations, des régularités pouvant mettre en évidence de nouvelles caractéristiques, de nouvelles causes à effets, voire 
de nouvelles découvertes. L'enjeu est fortement exprimé dans le cadre du consortium européen qui travaille sur le cerveau et qui envisage de rassembler toutes les connaissances décrites dans les jeux de données et les textes publiés. Les débats sont actuellement vifs entre éditeurs et chercheurs sur ce droit au «mining $»^{21}$.

Qu’en est-il pour les SHS ? Va-t-on assister à un déluge de données numériques comme pour les sciences de la nature dans les prochaines années? Il est probable que les objets connectés vont permettre de mesurer les échanges, les déplacements, les consommations des individus, etc. Mais ces traces numériques laissées par les humains vont-elles devenir l'enjeu central des activités scientifiques en sciences sociales? Lapproche quantitativiste prendra-t-elle le pas sur les approches qualitatives? On peut en douter et entrevoir plutôt une possible complémentarité de ces méthodes. Un autre type de données «moins brutes », et plus élaborées, plus qualifiées pour les sciences sociales, concerne les grandes enquêtes nationales telles que celles de l'INSEE, du Ministère de la Culture ou de l'OCDE. Elles ont un spectre assez large et une qualification institutionnelle suffisante pour susciter l'intérêt des chercheurs et donner lieu à de nouvelles investigations. En France, le réseau Quetelet ${ }^{22}$ organise déjà l'accès à de grandes enquêtes, à des recensements et autres bases de données issues de la statistique publique française, de la recherche. Louverture des données publiques devrait permettre de multiplier ces sources.

Mais, par contre, quel intérêt doit être porté à des données collectées dans le cadre d'une investigation spécialisée, répondant à certaines hypothèses propres à la recherche conduite? Peut-on en pareil cas obliger un chercheur à livrer ses données, alors même que sa motivation sera d’autant plus faible qu'il n’aura pas encore exploité ses données au mieux pour ses propres publications? Se pose aussi la question de la pertinence d'une réutilisation des données et de leur intelligibilité hors contexte. En l'absence d'une connaissance précise des conditions de leur collecte et d'explications de lecture, toute ré-exploitation des données est impossible. Faire parler des données sans contexte n’a pas de sens...

21 Voir Van Noorde Richard, 2014, «Elsevier opens its papers to text-mining», Nature, 506-17, 6 February: <http://www.nature.com/news/elsevier-opens-its-papers-to-text-mining-I. I4659>.

22 Voir <http://www.reseau-quetelet.cnrs.fr/>. 
Ajoutons qu'une autre limite à l'ouverture des données en SHS est posée par l'exigence éthique liée au respect de la confidentialité des données et la protection de la vie privée qui ne se pose pas de la même façon pour les sciences de la nature.

On peut donc douter que le potentiel de réutilisation des données en SHS soit à la hauteur de l'espérance politique forgée sur l'exemple de la génomique, adossée à de gigantesques flux de données numériques et dont l'enjeu reste le traitement algorithmique.

Un autre volet de l'ouverture des données concerne la disponibilité des données associées à une publication, étayant les résultats scientifiques avancés. Cet objectif est parfaitement fondé quand l'article est de nature empirique, car il peut renforcer la confiance et la qualité de l'expertise. Le gain qualitatif a été mis en lumière à l'été 20I3, avec l'exemple emblématique de l'article en économie ayant abusivement corrélé croissance, PIB et dette d'un pays. L’accès à la feuille de calcul Excel sur laquelle se fonde la démonstration a ainsi permis à trois économistes de l'université du Massachusetts de mettre à jour des manipulations dans les données invalidant les résultats annoncés ${ }^{23}$.

Le mouvement d'ouverture des données peut donc être situé en amont de la recherche (mettre à disposition des réservoirs de données brutes) ou en aval au moment de la publication (augmenter la qualité de l'expertise). Les objectifs ne sont pas identiques mais tous s'amalgament souvent de façon indifférenciée au sujet de l'«open data scientifique ». Pour les SHS, le modèle du réservoir amont de données brutes coopératif reste moins convaincant que pour les sciences de la nature. Quant à revendiquer l'accès aux données associées à la publication, c'est certes légitime mais de telles pratiques renvoient cependant à des méthodologies empiriques moins plébiscitées en SHS que dans les sciences de la nature. Il faut donc là aussi nuancer les enjeux.

23 Voir «Pour une recherche reproductible, publiez vos codes et données», Le Monde, 7 juillet 2013: <http://www.lemonde.fr/sciences/article/2013/07/15/pour-une-recherchereproductible-publiez-vos-codes-et-donnees_3447825_|650684.html>. 


\section{RISQUES POUR LES SHS}

Nous voudrions terminer par quelques recommandations, compte tenu des éléments apportés dans cette contribution, visant à promouvoir une transformation numérique soucieuse des valeurs fondamentales de la communication scientifique en SHS.

\section{I.L'IMPRUDENTE TRANSFORMATION DU MODÈLE DE L'ABONNEMENT}

Dans son étude menée avec huit associations américaines publiant des revues en SHS, Mary Waltham (2010) avait montré l'inadaptation du modèle de l'auteur-payeur pour ces revues ainsi que le risque associé à un embargo calqué sur les STM. En déplaçant les recettes de l'aval (abonnements) vers l'amont, l'OA fait dépendre fortement le fonctionnement des revues en SHS d'une logique de subventions publiques (ou de mécénat) car, contrairement à la majorité des champs en STM, il est difficile de croire que des fonds seront disponibles pour payer les frais de publication, en sorte que le modèle ne pourra en tout cas n'être que partiel. La liberté de publication d'un chercheur en SHS semble en totale contradiction avec la nécessité de payer pour s'exprimer. Comment en effet solliciter des fonds de son institution pour publier, quand il s'agit dans bien des cas de critiquer les institutions? L'histoire de l'édition en SHS s'inscrit à cet égard dans une volonté d'indépendance, de libre expression de cercles de chercheurs ayant souvent participé à la création des maisons d'édition qui animent encore la vie des idées aujourd'hui.

La transformation du modèle de l'abonnement en SHS semble imprudente car, contrairement aux STM, aucun modèle de substitution ne semble a priori viable, sauf à envisager une édition exclusivement publique ou financée à la bonne volonté d'un riche mécène... Est-ce un tel avenir qui doit être plébiscité ? Certains misent sur une troisième voix propre à l'économie de l'Internet qui voudrait concilier libre accès et recettes pour l'éditeur, à savoir des scénarios inspirés du modèle «freemium » faisant coexister deux versions d'un même bien, l'une gratuite accessible par tous et l'autre payante et associée à des services à valeur ajoutée devant déclencher l'achat de la 
version payante auprès de la base d'utilisateurs de la version gratuite. Ces modèles sont en test ${ }^{24}$ et sont encore peu probants dans un contexte où la propension à acheter est très faible et la satisfaction engendrée par la version gratuite suffisante dans bien des cas.

\subsection{L'EMBARGO, LA CLÉ DE VOÛTE DE L'ÉQUILIBRE}

Si la voie verte paraît plus conciliable avec l'édition en SHS, il faut encore en définir les règles, et donc la durée de l'embargo, délai qui s'impose entre la date de publication et la date de mise en accès libre de cette publication. Des mesures trop agressives pourraient être fatales à de nombreuses revues et conduire à un appauvrissement du paysage éditorial. L'exemple britannique est emblématique de cet empressement : favorable à la voie dorée tout en reconnaissant que la voie verte pouvait parfois être préférée, le Research Councils UK avait commencé par fixer l'embargo à 12 mois pour les SHS. Ce délai a été étendu ensuite à 24 mois à la condition que la modalité dorée soit proposée et sous réserve que les fonds ne soient pas disponibles pour les auteurs ${ }^{25}$. La politique adoptée demeure en test pendant 5 ans et sera réexaminée périodiquement.

En France, les revues ont été encouragées, sur la plate-forme Héloïse ${ }^{26}$, à faire connaître l'embargo qui leur semblait conciliable avec leur modèle économique. En mars 20I3, tout domaine confondu, lorsque les éditeurs autorisaient le dépôt de l'article, l'embargo était majoritairement fixé à 3 ans (Dillaerts, Chartron, 20I3). Pour les SHS, les données relatives aux barrières mobiles en vigueur pour les 370 revues de la plate-forme Cairn.info (voir Figure 4) sont plus significatives: la majorité des revues (85\%) ont fixé des délais à 3 ans et plus. Les délais les plus fréquents sont de 3 ans (36\%) et de 5 ans (26\%), ce qui est bien supérieur aux I2 mois envisagés par la CE.

24 Modèle Freemium d'OpenEdition: <http://www.openedition.org/8873?lang=fr>.

25 RCUK Policy on Open Access and Supporting Guidance:

<http://www.rcuk.ac.uk/RCUK-prod/assets/documents/documents/RCUKOpenAccessPolicy.pdf>.

26 Voir <http://heloise.ccsd.cnrs.fr/>. 
Figure 4: Délais d'embargos sur Cairn.info

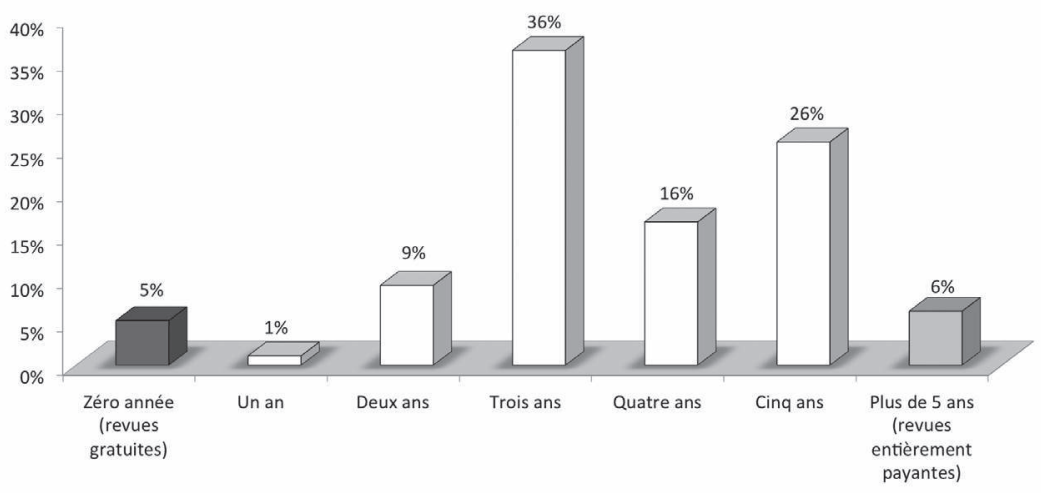

On comprend mieux cette prudence vis-à-vis du libre accès quand on observe les données statistiques de consultation fournies par cette plateforme $^{27}$ (voir Figure 5). Ainsi il apparait faux de penser qu'en SHS les i2 ou les 24 derniers mois concentrent la majorité des accès. Dans le cas de cette plate-forme, les I2 derniers mois cumulent uniquement i7,9\% des consultations. La demi-vie des articles (plus de 50\% des consultations) est atteinte avec les 4 dernières années de publication. Un embargo trop court risquerait en conséquence très certainement de susciter de nombreux désabonnements. Cette spécificité des SHS pour les durées d’embargo a par ailleurs été reconnue par l'association Science Europe (20I3) qui fait aussi le constat de l'inadéquation de la voie dorée pour ces domaines.

27 Voir <http://www.openaccess-shs.info/open-access-quelle-duree-dembargo-pour-quellespublications-le-cas-des-sciences-humaines-et-sociales/>. 
Figure 5: Répartition des consultations sur Cairn.info

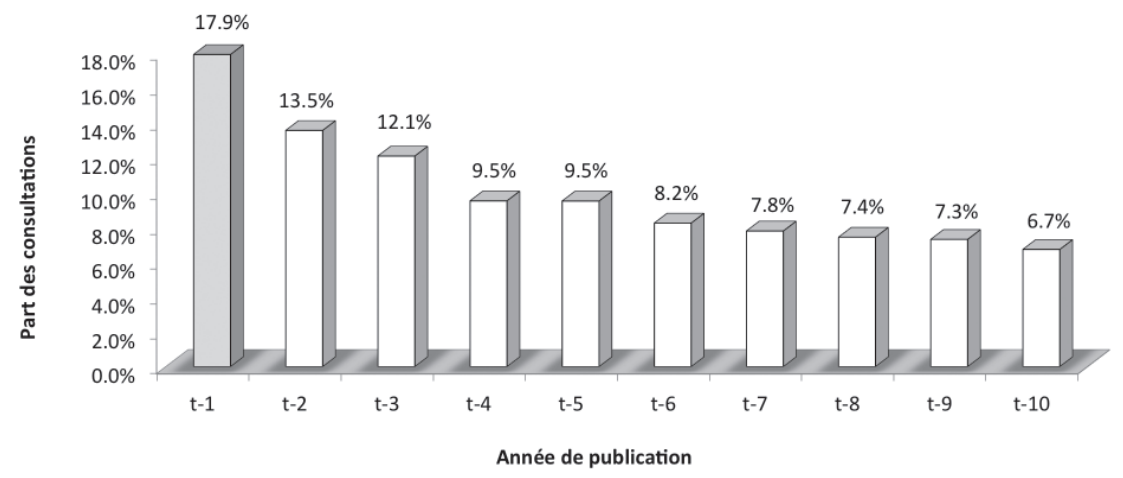

\section{CONCLUSION Scénarios et principe de précaution}

Si l'on peut penser que les éditeurs internationaux qui captent l'audience internationale peuvent réussir à concilier OA et édition en SHS sur des produits phares et sur des revues largement partagées par certaines communautés en SHS - pour lesquelles des financements pourront être trouvés car elles sont internationales et considérées comme prestigieuses -, il n'en va pas de même pour la majorité des acteurs qui contribuent à la richesse et à la diversité des publications en SHS.

Comme scénario final, nous voudrions attirer l'attention sur la disparition potentielle d'une frange de l'édition en SHS. Nous faisons l'hypothèse que la fragilisation de nombreux acteurs nationaux non financés pleinement par l'État conduira à un double mouvement de concentration : une concentration sélective opérée par les plates-formes internationales en STM qui feront «leur marché» sur les publications en SHS et les intégreront à la marge dans leur offre, et une concentration inflationniste de titres vers des plates-formes de financement public peu sélective. La politique nationale menée sera donc déterminante sur la régulation qui est souhaitée. 
Si l'objectif majeur est de parvenir à un délai d'embargo le plus court possible, il faudra alors envisager des financements compensatoires pour les éditeurs non publics, au risque sinon de les voir disparaitre sur ce créneau de l'édition de recherche en SHS. L’Allemagne a ainsi engagé plusieurs fonds d’aides à la publication en Gold OA pour l'édition nationale y compris.

Si l'objectif majeur est avant tout de raisonner sans aucun financement supplémentaire pour l'accomplissement du libre accès, le principe de précaution devrait conduire à un aménagement plus large de la période d'embargo que celui proposé par la Recommandation européenne, si l'intention est bien aussi de préserver l'édition nationale.

Si l'objectif est de promouvoir uniquement l'édition selon une économie publique, et de centraliser les lieux de diffusion, on atteindra alors l'OA plus rapidement mais en exposant le modèle éditorial à une insécurité permanente quant à la pérennité des subventions. En outre, en l'absence d’une sélection qualitative en amont procédant de l'acte d'abonnement, on entrerait dans une logique où la valeur des biens d'information rendus accessibles ne serait plus a priori garantie par un acheteur.

Enfin, on peut aussi envisager un autre scénario selon lequel l'argent que libérera le développement du modèle auteur-payeur en STM dans les budgets des bibliothèques, sera pleinement consacré aux SHS qui n’ont jamais connu les dérives inflationnistes des STM, et dont la valeur éditoriale est beaucoup plus distribuée, laquelle doit beaucoup à la dynamique intellectuelle qui s'installe entre les éditeurs et leurs auteurs. Au-delà de l'OA, la qualité du projet éditorial doit rester la priorité pour la diffusion des SHS. 


\section{BIBLIOGRAPHIE}

ALPSP, 2012, The potential effect of making journals free after a six month embargo, A Report for the Association of Learned, Professional and Society Publishers and The Publishers Association: <http://www.alpsp.org/Ebusiness/Aboutalpsp/ ALPSPStatements/StatementDetails.aspx?ID $=407>$.

CHARTRON G., 2003, «Éléments pour une approche comparée», in Colloque du centre de coopération interuniversitaire franco-québécois "La communication scientifique: enjeux du partage de la connaissance", 4-6 juin, Montréal: $<$ http://archivesic.ccsd.cnrs.fr/sic_00000435.html>.

-, MINON M, 2005, Analyse comparée de l'offre des revues universitaires de sciences humaines et sociales en France, en Espagne et en Italie, Étude réalisée pour le Ministère de l'Éducation nationale de l'Enseignement supérieur et de la Recherche: <http://archivesic.ccsd.cnrs.fr/sic_0000156I.html>.

CHESBROUGH H., 2003, Open innovation: The new imperative for creating and profiting from technology, Boston, Harvard Business School Press.

DILLAERTS H., CHARTRON G., 2013, «Héloïse: towards a coordinated ecosystem approach for the archiving of scientific publications?», Learned Publishing, 26-3, p. 173-179, doi: <http://dx.doi.org//0.1087/20130304>.

COLLECTIF, 2013, «Open Access: le travail scientifique en sciences humaines et sociales et le débat public fragilisés par les mesures préconisées par la Commission européenne», Paris: <http://www.openaccess-shs.info/motion/>.

EUROPEAN COMMISSION, 2006, Study on the economic and technical evolution of the scientific publication markets in Europe: <http://ec.europa.eu/research/ science-society/pdf/scientific-publication-study_en.pdf>.

GFIl, 2009, L'Édition scientifique française en sciences sociales et humaines, 3 volumes: <http://www.gfii.fr/frl document/l-edition-scientifique-francaise-en-sciences-sociales-et-humaines>.

HARNAD S., 1990, «Scholarly Skywriting and the Prepublication Continuum of Scientific Inquiry», Psychological Science, I, p. 342-343: <http://cogprints.org//58I/>. 
KLING R., MCKIM G., 2000, «Not Just a Matter of Time: Field Differences and the Shaping of Electronic Media in Supporting Scientific Communication », Journal of the American Society for Information Science, 51-14, p. 1306-1320: <http://xxx.lanl.gov/ftp/cs/papers/9909/9909008.pdf>.

MINON M., 1990, «L'État de l'édition en sciences humaines et sociales», Cahiers de l'économie du Livre, 2, 1990, Éditions du Cercle de la Librairie.

OCDE, 2007, Principes et lignes directrices de l'OCDE pour l'accès aux données de la recherche financée sur fonds publics, Éditions OCDE, doi: <http://dx.doi. org/l0.1787/9789264034020-en-fr>.

-, 2012, «Open science», in OECD Science, Technology and Industry Outlook 2012, Éditions OCDE, doi: <http://dx.doi.org/l0.1787/sti_outlook-2012-21-en>.

-, 2013, Commercialising Public Research: New Trends and Strategies, Éditions OCDE, doi: <http://dx.doi.org//0.1787/978926419332I-en>.

ODLYZKO A. M., 1995, «Tragic loss or good riddance? The impending demise of traditional scholarly journals », International Journal of Human-Computer Studies, 42-I, p.7I-122: <http://www.jucs.org/jucs_0_0/tragic_loss_or_good>.

OKERSON A.S., O'DONNELL J.J., 1995, Scholarly Journals at the Crosroads: A Subversive Proposal for Electronic Publishing, ARL Editions.

PENIN J. et al., 2011, «New shapes and new stakes: a portrait of open innovation as a promising phenomenon », Journal of Innovation Economics, 7-I, p. II-29, doi: <http://dx.doi.org/|0.3917/jie.007.0011>.

ROYAL SOCIETY, 2012, Science as an open enterprise: <http://royalsociety.org/uploadedFiles/Royal_Society_Content/policy/projects/sape/2012-06-20-SAOE.pdf>.

SCIENCES EUROPE, 2013, Open Access Opportunities for the Humanities, Humanities Committee: <http://www.scienceeurope.org/uploads/ PublicDocumentsAndSpeeches/SE_Humanities_Paper_FIN.pdf>.

VINCENT N., WICKHAM C. (dir.), 2013, Debating Open Access, British Academy: <http://www.britac.ac.uk/openaccess/debatingopenaccess.cfm>.

WALTHAM M., 2010, «Humanities and social science journals: a pilot study of eight US associations» Learned Publishing, 23-2, p. 136-143, doi : <http://dx.doi. org/10.1087/20100209>. 
